\title{
Markovian Analysis of Phasic Measures of REM Sleep in Normal, Depressed, and Schizophrenic Subjects
}

\author{
Alan B. Douglass, Kathleen Benson, Elizabeth M. Hill, and \\ Vincent P. Zarcone, Jr.
}

Rapid eye movement (REM) phasic activity refers to brief events that occur in periods of REM sleep, such as individual eye movements (EMs). REM density (RD) is the bestknown measure of such activity, although reports of $R D$ differences among normal, depressed, and schizophrenic subjects have been equivocal. $R D$ is a measure with a large variability, and its physiological substrate is not known. We sought a more consistent measure which might also suggest the underlying physiology. Using the time intervals between individual EMs, we calculated empirical probability distributions which showed that EMs fell into two subgroups or states: "burst" and "isolated." Then, a novel Markov chain model of sequential transition between the states was calculated for nine normal, eight schizophrenic, and seven depressed male veterans. A significantly higher probability of remaining in the burst state was observed in both patient groups. The actual number of EMs in the isolated state was nearly identical in the three groups. Possible pontine neurochemical explanations involving cholinergic and serotonergic mechanisms are discussed.

\section{Introduction}

Abnormalities of sleep architecture have been noted in several psychiatric syndromes when compared with normal controls. These include decreased slow-wave sleep (Benson and Zarcone 1989; Kupfer et al 1984), shorter REM sleep latency (Kupfer 1976, Keshavan et al 1990), and increased "REM phasic activity," that is, the amount of eye movement activity in REM sleep (Reich et al 1975). Though initial reports showed that many of these abnormalities were found only in patients with major depressive disorder, more recent findings suggcst that schizophrenics have similar ahnormalities (Zarcone et al 1987).

Of the above abnormalities, REM phasic activity is reported on the least, perhaps because of the need for more complicated data unalysis. Simple counts of the number of

From the Psychiatry Service, Veterans Administration Medical Center, Palo Alto, and the Department of Psychiatry and Behavioral Sciences, Stanford University School of Medicine, Palo Alto, California (KB, VPZ); and the Ann Arbor VA Medical Center Psychiatry Service, and Department of Psychiatry, University of Michigan, "nn Arbor, Michigan (ABD,
EMH). Address reprint requests to Dr. Douglass, c/o Psychiatry Service 116A, Ann Arbor VAMC, 2215 Fuller Road, Ann Arbor,
MI, 48105.

Received February 14, 1991; revised September 19, 1991 
EMs are inadequate as the curation of a REM sleep period varies consideraibiy even in a single individual. One solution has been the calculation of a "REM eye movement density" measurement, the number of EMs per unit time in a period of REM sleep. This is often called simply "REM density" (RD), and has been calculated by various methods. Evidence of RD differences between groups of psychiatric patienis has been equivocal.

Feinberg et al (1964) found that hallucinating schizophrenics had a RD similar to normals, but that nonhallucinating schizophrenics had a significantly lower RD than either group. Reich et al (1975) observed RD to be significantly higher in schizoaffective patients compared with acute and "latent" schizophrenics. Gillin and Wyatt (1975) found no difference between the RD of schizophrenic and normal controls. Although Foster et al (1976) demonstrated that RD of the first REM sleep period separated primary from secondary depressives, Thase et al (1986) could not replicate the finding. Rather, they found that RD correlated with severity of depressive illness. Reynolds et al (1983) reported RD in narcoleptics to be significantly higher than even the RD of depressed patients. Kempenaers et al (1988) found a nonsignificant trend for RD in schizophrenics to be higher than normals and lower than depressed patients. Benson and Zarcone (1991) found that RD did not differ significantly between depressed and schizophrenic subjects. These authors also showed that in normals, Zung depression scores were positively correlated to RD (Zarcone and Benson 1983).

However, even RD is a rather global measurement. It represents only an average of the underlying pattern of clumping or bursting of the EMs within a REM period. To get at the deeper information, one must measure the time interval between successive EMs, hereafter called the "interrapid EM interval" (IRI).

Unfortunately, conventional statistics are not well suited to IRI data. One older approach was to submit counts of EMs per unit time (not IRIs) to time-series analyses such as auto-correlation, Fourier transform, or period analysis (Krynicki 1975; Lavie 1979), but some assumptions of time-series analysis are not met by such data (Ktonas 1974). Two issues are relevant: the statistical frequency distributions of the IRIs, and the "stationarity" of the process that generates IRIs-whether the generating process is constant over time, or varies in ways that would produce a nonrandom trend. The fact that IR Is occur in "bursts" between which there are many "isolated" IRIs violates the stationarity assumption of conventional time-series analysis. Regarding statistical distribution, Boukadoum and Ktonas (1988) fitted different linear functions to burst and isolated IRIs displayed on a semi-log probability distribution, which suggested that the two categories of IRI, burst versus isolated, may be generated by separate physiological processes. A probability distribution can be approximated by a frequency histogram of the IRIs of one REM period from one person, divided by the total number of IRIs in that REM period.

The novel Markovian statistical approach of Boukadoum and Ktonas (1988) was designed to remedy the shortcomings of conventional time-series analysis of IRIs. The method as yet has only been reported for normal subjects, but it shows considerable promise as a research tool in psychopathology, which the present article explores. It could be described as a categorical time-series approach, the categories being burst and isolated IRIs. The probabilities of transition from one category to the other are calculated from the sequential appearance of IRIs throughout a REM period. Though burst density or isolated density might be calculated in the manner of RD, neither demonstrates the sequence in which an IRI in burst succeeds an isolated IRI in the way that the Markovian analysis does.

The development of this Markovian method began in the 1970s, and is not to be 
confused with Makovian methods of predicting the transition from REM sleep to other sleep stages (Kemp and Kamphuisen 1986). Ktonas and Smith (1976) were the first to suggest that a Markov model might be applied to the transition between burst and isolated IRIs within a single REM period. Ktonas and Bonilla (1979) stated the mathematical assumptions underlying a Markovian analysis of IRIs. Briefly, they employed a firstorder Markovian model with two dichotomized (quantitized) states: IRIs less than 5 sec (burst IRIs) and IRIs greater than 5 sec (isolated IRIs). A numerical example in Ktonas and Boukadoum (1987) shows how this approach can find differences between two REM periods having identical REM densities. These authors also estimated the theoretical sampling distribution of a parameter " $C$ " derived from the Markcvian state transition probability matrix (STPM) using a Monte Carlo simulation (Ktonas et al 1981). This siriulation led the author to suggest the minimum number of IRIs in a REM period required for a valid Markovian calculation (e.g., 70 IRI for a $5 \times 5$ transition matrix, see below). Further elaboration by Jansen and Cheng (1988) showed that one requires, for an accurate calculation, $5 n^{2}-8 n^{2}$ IRI, where $n$ is the number of rows in the square transition matrix. For example, a $2 \times 2$ matrix would require 20-32 IRIs.

Boukadoum (1983), Boukadoum and Ktonas (1988) showed that the "stationarity assumption" for Markovian analysis of REM sleep was met if the REM period was at least $8 \mathrm{~min}$ long. They also found that the STPMs have a stationarity (do not differ significantly) between the several REM periods of a single night. This is in contrast to the well-known increase in RD and REM period duration towards morning.

The above techniques have never been used to analyze the REM sleep of psychiatric patients. We applied IRI statistical frequency histograms and Markovian analysis of IRI burst-isolated transitions to the REM sleep of schizophrenic, depressed, and normal subjects. Because conventional REM phasic activity measures such as RD seem to show inconsistent differences between depressed and schizophrenic subjects, we wondered if this novel statistical analysis would show a difference. We thus sought to disprove the null hypothesis: The Markovian patterns of IRIs in schizuphrenic and depressed patients versus normals do not differ significantly.

\section{Methods}

\section{Patient Selection}

We studied 24 subjects, all male veterans; 15 were patients from the Palo Alto VA Medical Center's Psychiatry Service. All were inpatients in the Clinical Research Center, and were diagnosed using Research Diagnostic Criteria (RDC) (Spitzer et al 1978). Seven had major depressive disorder (DEPR) and eight were schizophrenic (SCHZ). In addition, nine normal male veteran paid volunteers (NORM) were recruited from the community to control for socioeconomic and educational factors. All were in good health and had no problems sleeping. None abused alcohol or illicit drugs. They were screened for psychopathology using the Structured Clinical Interview (SCI) of Burdock and Hardesty, which provides 10 indices of psychiatric disorder. None of the controls exceeded the threshold on any of the 10 subscales.

There was a significant difference in the mean age of subjects, likely explained by the differing peak age of onset for depression and schizophrenia (mean \pm SD: NORM $=$ $27.3 \pm 7.3, \mathrm{DEPR}=40.0 \pm 13.9, \mathrm{SCHZ}=28.3 \pm 2.5$, analysis of variance (ANDVA) $F=5.01, p=0.015$ ). 
With the exception of chloral hydrate PRN, all patients were free of psychotropic medications for a minimum of 2 weeks before the all-night polysomnographic recordings. In all cases, no chloral hydrate was given in the $\mathbf{7 2} \mathrm{hr}$ prior to the study. Following two adaptation nights, data were obtained from two consecutive recording nights to assess reliability of the measured variables.

\section{Procedures}

Using a Grass Polygraph, electroencephalogram (EEG), chin electromyogrcn (EMG), and electrooculogram (EOG) were recorded on both nights. Sleep stages were scored according to the conventions of Rechtschaffen and Kales (1968). The EOG technique was a differential recording between pairs of shunted electrodes attached to the inner and outer canthi of opposite eyes (Hord 1975). Grass Polygraph filter settings for EOG were $30 \mathrm{~Hz}$ high-filter, $0.3 \mathrm{~Hz}$ low-filter, $50 \mu \mathrm{V} / \mathrm{cm}$ gain. Data were simultaneously recorded on an AMPEX FM tape recorder for off-line computer analysis via the Grass J6 output. Digitization of the raw data and computerized waveform analysis were performed using the REMDTEK software (Schreier et al 1977) with parameters set to accept a waveform as an EM if its onset exceeded $25 \mu \mathrm{V}$ within $200 \mathrm{msec}$. Any IRI of less than $60 \mathrm{msec}$ was assumed to be artifactual and was discarded.

\section{Statistical Analysis}

Boukadoum and Ktonas (1986) suggested using a 200 msec refractory period after the detection of each EM, to avoid mis-identifying amplifier artifacts as EMs. The $200 \mathrm{msec}$ rule is not universally used as it depends on the filter settings used on the polygraph amplifier (our own system used a 60 msec rejection rule). Yet, we wished to compare our findings with those of Boukadoum and Ktonas (1988). We therefore wrote a program that summed any IRIs in our raw data within $200 \mathrm{msec}$ after a given IRI, and added the time to the next one. This procedure was repeated until the minimum IRI was $200 \mathrm{msec}$. This approximation provided the fairest comparison of our data with theirs, and is shown in Table 2 (middle section). Our raw data were used for all other calculations, including Figure 1, the CATMOD procedure, and the results in Tables 3-5.

Ten conventional measures of activity were calculated for each KEM sleep period: (1) number of IRIs (\#IRI); (2) number of minutes of REM sleep (\#MIN); (3) REM density (RD = \#IRIs/\#MIN); (4) number of IRis in bursts where burst IRIs were less than $2.0 \mathrm{sec}$ apart, expressed per hr of REM sleep (BURIRI/HR) (Note that this definition of a burst differs from the "less than 5 sec" definition in the Markovian analysis section. The reason for reporting 2-sec bursts is to allow comparison with previous research reports where this definition was commonly used); (5) percent of \#MIN spent in bursts (BUR\%TM); (6) number of isolated IRIs per hr of REM sleep (ISOLIRI/HR); (7) percent of REM sleep time spent as isolated IRIs (ISOL\%TM); (8) number of IRIs per 2-sec burst (IRI/BUR); (9) the number of bursting episodes detected per hr of REM sleep (BURCNT/HR); and (10) a measure of the amount of fragmentation (FRAG) of REM sleep by other sleep stages, where $100 \%$ refers to unbroken REM sleep. As these measures were likely to be intercorrelated, a nonparametric intercorrelation matrix (Spearman's Rho) was calculated. Those found to be least correlated to others (variables 2 and 10), and three relating to burst-, or isolated-RD (variables $1,4,6$ ) were submitted to ANOVA: group $\times$ night $\times$ REM period, with Bonferroni comparisons of means post hoc. Three-way ANOVAs were 
initially performed, but the NIGHT variable was never significant. Accordingly, 2-way ANOVAS were done, pooling both nights together. Statistical Analysis System (SAS) version 6.03 General Linear Model (GLM) procedure was used.

The frequency and probability distributions of the IRIs measured in these three groups have already been published (Douglass et al 1985). The data were replotted in the manner of Boukadoum and Ktonas (1988) using semi-log axes. Linear regressions were done to estimate the burst and isolated IRI probability density functions (PDFs).

The Markovian analysis proceeded as follows: the transition between burst and isolated IRIs required a two-state model $(2 \times 2$ raw transition matrix, RTM) where state 1 represented IRIs quantitized as less than $5 \mathrm{sec}$ and state 2 represented IRIs greater than $5 \mathrm{sec}$. The matrix was filled using frequency counts $(n)$ of transitions between the two quantitized states. A numcrical example is shown in Table 1. This RTM was then converted into a maximum-likelihood estimate of the STPM for each REM sleep period (from Boukadoum 1983):

$$
\hat{P}_{i j}=\frac{n_{1 j}}{n_{1}}
$$

[equation 1]

where

$\hat{P} \quad$ is the estimated STPM

$n_{i j}$ is the frequency count of the cell of the RTM, row $i$ column $j$

The sum of probabilities in each row of this matrix is 1.00 , by definition. Also generated was the "state probability" for each of the two states, which is the likelihood of each state occurring in that REM sleep period, irrespective of order of appearance.

A statistical test was suggested by Boukadoum (1983) to determine significant differences between pairs of STPMs. $A \chi^{2}$ value is obtained with dfs defined by the dimensions of matrices involved:

$$
\chi^{2}=\sum_{i=1}^{N} \sum_{j=1}^{N} \frac{n_{i}\left(\hat{P}_{y}-\dot{P}_{i j}\right)^{2}}{\dot{P}_{y j}}
$$

where

$$
\begin{aligned}
N & =\text { number of rows in STPM matrix } \\
\mathrm{df} & =\text { degrees of freedom }=N(N-1) \\
n 1_{1} & =\text { row frequency count, row } i \text { of } \cdot \text { STPM } \\
n 2_{1} & =\text { row frequency count, row } i \text { of } \cdot \text { STPM } \\
n_{1} & =\text { SQRT }\left(n 1_{1} \times n 2_{1}\right) \\
\hat{P} & =\text { STPM predicted from observed data } \\
\dot{P} & =\text { any other STPM to which } \hat{P} \text { is compared. }
\end{aligned}
$$




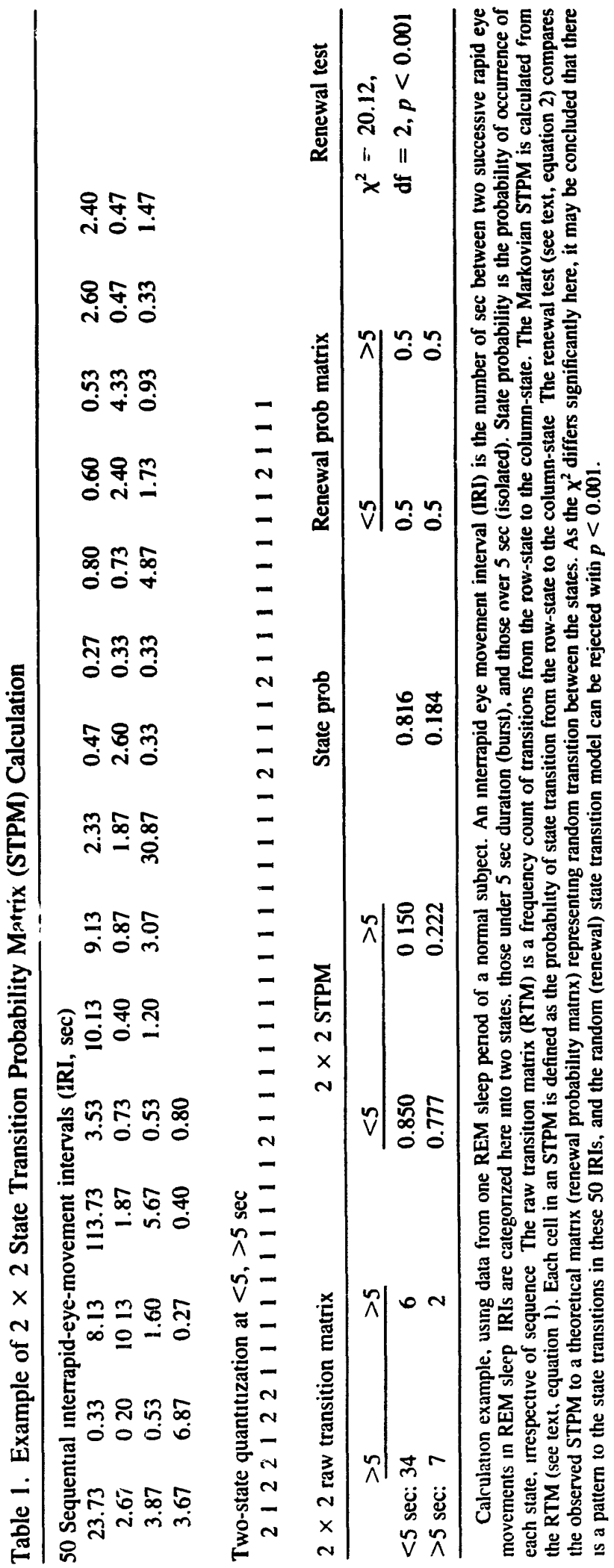


A special case is the comparison of an experimental STPM against a "renewal process" STPM representing random transition. If significant, this comparison indicates that the experimental STPM shows patterned transition between states and does not correspond to a renewal (purely random) process.

$2 \times 2$ STPMs were calculated for each REM period that was over $8 \mathrm{~min}$ long and which had over 75 IRIs (see CATMOD below). Each STPM was then compared to the theoretical "renewal matrix" using equation [2], and a tally was made of how many REM periods differed from a renewal model at a $\chi^{2} \geq 9.2$, $p<0.01$, $\mathrm{df}=2$.

As the RTM frequencies underlying the STPMs of each REM sleep period are essentially $2 \times 2$ contingency tables, the question arises of what statistical test to use on them in a factorial experimental design such as the present one. The solution of Boukadoum and Ktonas (1988), reponing on a single group, was to perform a nonparametric test on the STPMs from the REM periods of one night for each subject in order to demonstrate stationarity. But, due to the small number of REM periods in one subject night, true differences between REM periods across subjects could be missed due to type II error. Though this method is both conservative and valid, it does not provide a statistical test for differences between experimental groups.

For a group summary, they reported the mean and SD of the 4-cell STPMs across all subjects for each REM period. We have generated a similar table from our data for the purposes of comparison (Table 2), but there is a problem with this approach: the mean is not the best measure of central tendency for samples from nonnormally distributed populations like proportions, as is the case with the STPMs. A maximum-likelihood estimate applied to pooled frequencies of all $2 \times 2$ tables would be a better estimate of the true group cell probabilities, or a logit transformation could be employed to normalize such a distribution (Snedecor and Cochran 1980). These methods would produce tables that better reflected the true group probabilities. This criticism applies only to the tabular display; the statistical tests employed by Boukadoum and Ktonas (1988) were appropriate.

The analysis of Markoviar dpca by log-linear models has been well described by Bishop et al (1975). Our solution employed the categorical log-linear model (CATMOD program, SAS version 6.03, SAS Institute, Cary, NC) which is designed for data of this type. CATMOD required a minimum of 75 data points (IRI transitions) to estimate a $2 \times 2$ table. The response profile for CATMOD was defined as the RTM frequency counts underlying the $2 \times 2$ STPM of each REM period. The logit method was used to estimate STPM probabilities in a full factorial design ( 3 groups $\times 2$ nights $\times 4$ REM periods $\times 2$ states-burst and isolated IRI). Individual subjects were not entered into CATMOD. Though a repeated-measures design on subjects would have been desirable, there was sufficient missing data to preclude this approach. It is possible to use raw frequency counts directly in CATMOD, but due again to the small number of subjects and substantial intersubject variation, we chose to estimate the group frequency count by squaring the mean of square-root-transformed individial frequencies (Snedecor and Cochran 1980). This method is more conservative than pooling frequency counts where subjects in a single group differ a great deal from one another.

CATMOD results are reported in a tabular form designed to resemble the familiar ANOVA table, except that $\chi^{2}$ values replace the $F$ ratios of ANOVA. The $\chi^{2}$ contribution of the classification variables group, night, and REM period and their saturated crossed effects were calculated. As the night factor was never significant, nor were group $\times$ night $\times$ REM period $\times$ state 3- and 4-way interactions, the final CATMOD model was reduced from a fully saturated factorial model to group $\times$ REM period $\times$ state main 


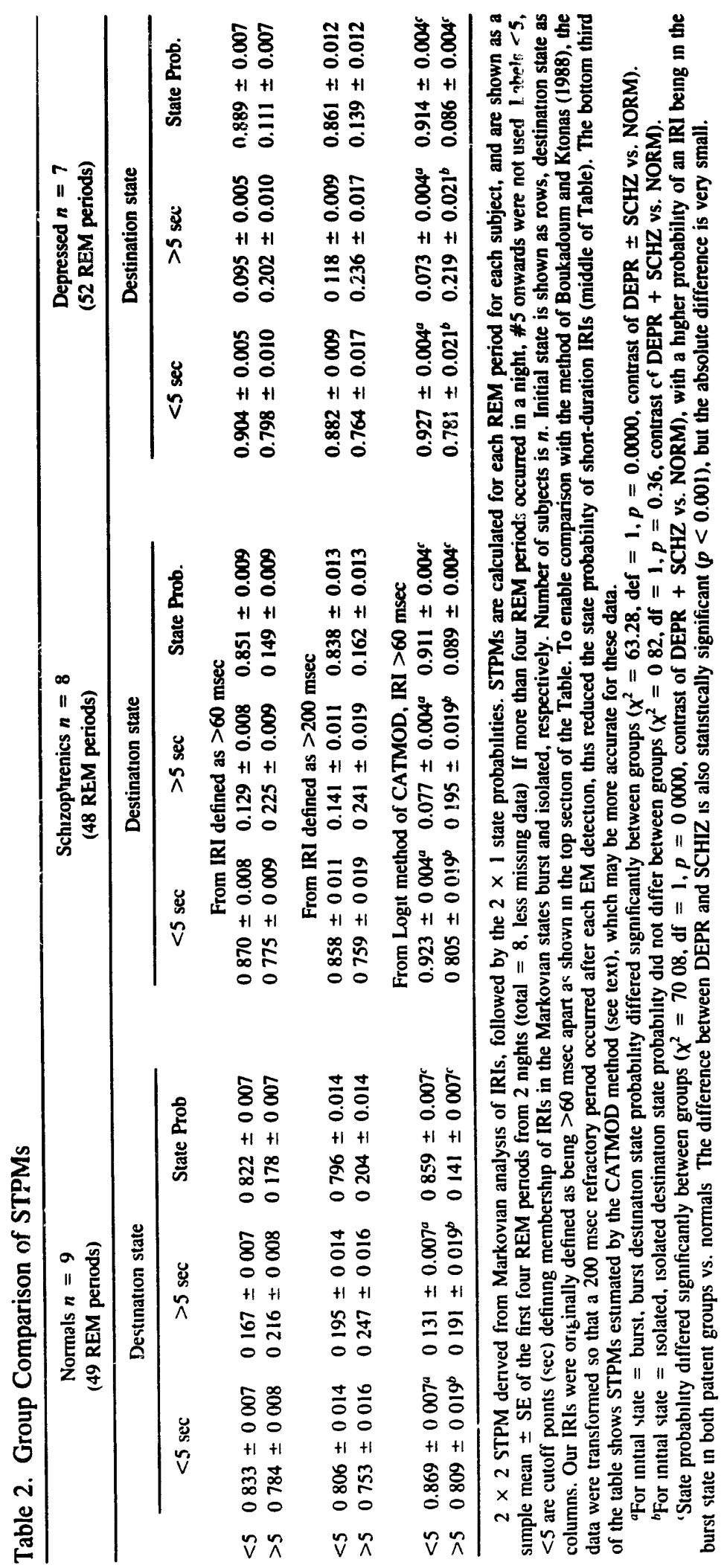


Table 3. Summary Table from Categorical Log-Linear Model Analysis (CATMOD)

\begin{tabular}{lccl}
\hline \multicolumn{1}{c}{ Source } & DF & $x^{2}$ & \multicolumn{1}{c}{$p$} \\
\hline Intercept & 1 & 1940.59 & 0.0000 \\
Initial-state (burst, isolated) & 1 & 114.01 & 0.0000 \\
Group & 2 & 7.75 & 0.02 \\
REM period (\#1, 2, 3, or 4) & 3 & 3.00 & 0.39 \\
Inittal-state X group & 2 & 16.65 & 0.0002 \\
Initial-state X REM period & 3 & 5.41 & 0.144 \\
Group X REM period & 6 & 16.35 & 0.012 \\
Residual & 6 & 4.13 & 0.659 \\
\hline
\end{tabular}

The dependent varlable in this analysis is destination state probability. The CATMOD table is designed te resemble an ANOVA table, but uses $\chi^{2}$ instead of $F$ ratios. These results indicate that the destination state is significantly ciependent on the initial state; i.e., there is a strong tendency for a given IRI to be a member of the same state as ine IRI that jreceded it. The three groups differ significantly on the probability of destination state; furthermore, this is of a significantly different pattern in different groups (initial state by group interaction). The significant group by REM period interaction indicates that groups also differ in their pattern of destination state probability over the four REM periods (see Table 4). The nonsignificant residual indicates a good fit of the statistical model to the observed data.

etfects and 2-way interactions only. It is the latter model that forms the basis of the tables. Adequate goodness-of-fit of the model resulted in a nonsignificant residual $\chi^{2}$ (Table 3 ). CATMOD was also used to estimate the STPMs for group $\times$ REM period (Table 4 ). Contrasts were used in a subsequent CATMOD to break down significant differences.

Results

In all, 181 REM periods were recorded. After removing those with fewer than 75 IRI, 149 remained. All subjects had STPMs that were significantly different from a renewal STPM with the exception of one REM period of one subject in the NORM group. This is in accord with Boukadoum and Ktonas (1988) who found $89 \%$ passed when a $5 \times 5$ STPM was used. Thus, transitions between the two states were not random.

Probability density function estimates resulting from a single-process model of IRI generation are shown with the raw IRI data in Figure la. Using the same raw data, linear estimates for the burst and isolated IRI density functions of a two-process model are shown in Figure $\mathrm{lh}$. The latter is a better physiological explanation. It also fully supports the Markovian assumption of a quantitization of IRIs into burst and isolated groups. Results for our NORM group closely matched those of Boukadoum and Ktonas (1988) (ses Figure 1 legend for numerical values).

In Table 2, STPM data are displayed by group. It can be seen that the raw data in the upper third of the table produce slightly higher values for the burst-to-burst transition STPM cell than do the data in the middle third which have been adjusted to remove IRI less than $200 \mathrm{msec}$. Overall, the pattern in the NORM group is similar to that reported by Boukadoum and Ktonas (1988) for normals. The top two-thirds of the table are calculated by Boukadoun's group arithmetic mean method, and the bottom third of the table shows CATMOD's logit estimates of the STPMs. The latter are the highest probabilities in the table, and also theoretically the best estimates of these probabilities. The groups differed significantly. Considering thi initial state "burst," the destination state transition probabilities of the DEPR and SCHZ groups were significantly higher than NORM group (top left cell) in a contrast. The DEPR and SCHZ groups also were 


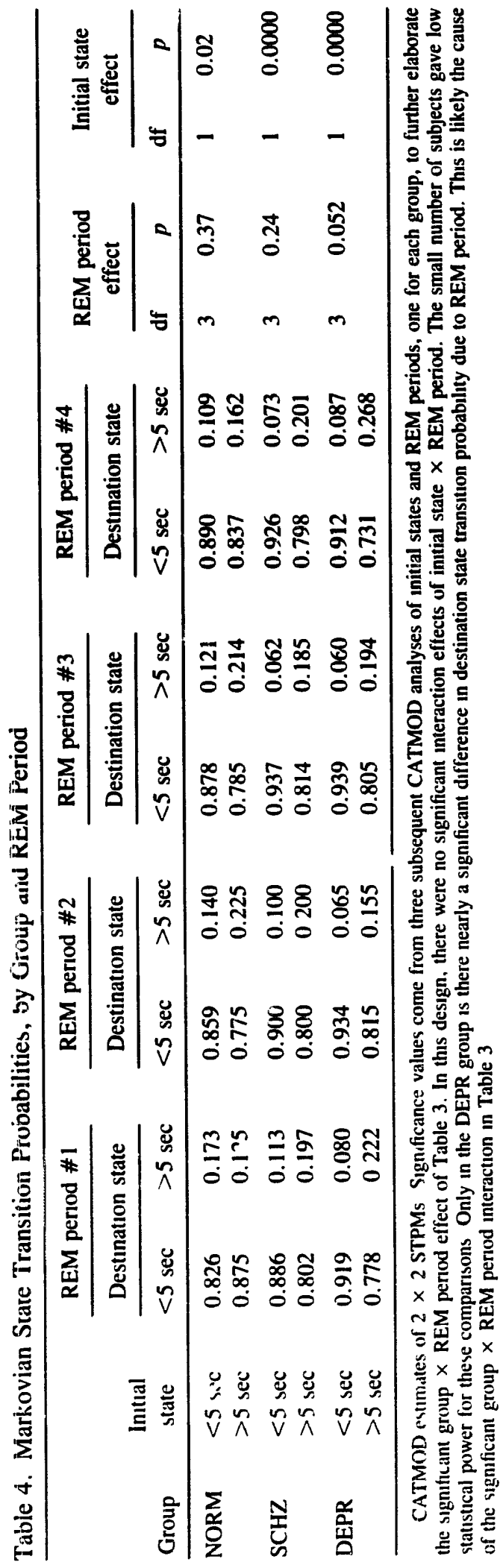




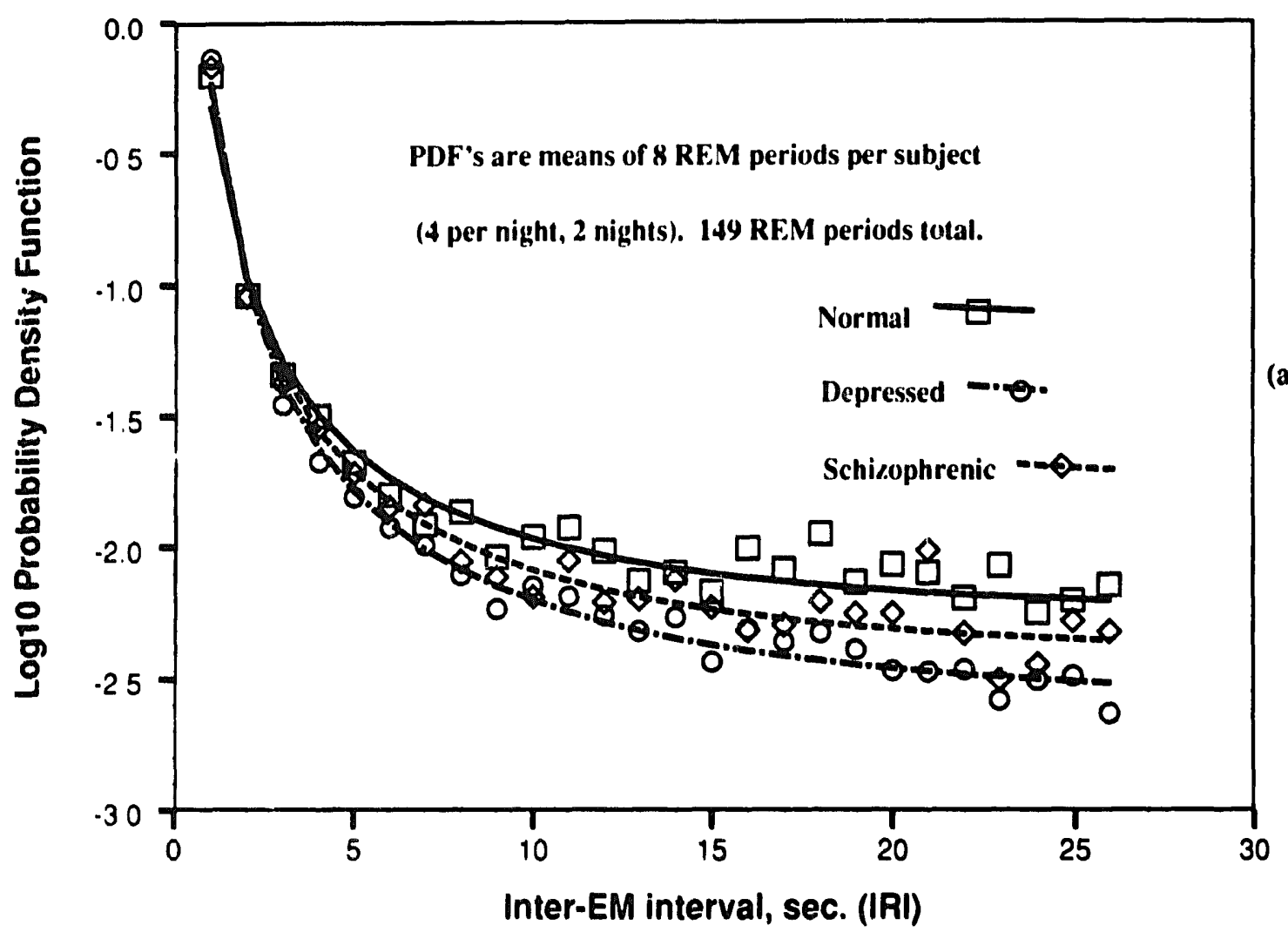

Figure 1.(a) Estimated probability density functone (PDF) of IRIs for the three groups, using a singleprocess model of eye-movement (EM) generation. Data are replotted from Douglass et al (1985). Singleprocess model requires an unusual nonlinear regression of logarithmic data which is difficult to explain physiologically.

statistically significantly different from each other, but the absolute difference was very small. Considering the initial state "isolated," the destination state transition probabilities did not differ significantly among the three groups.

The summary table from CATMOD is shown in Table 3. There was a significant effect of initial state, group, and state $\times$ group interaction. There was no significant REM period effect, but there was a significant group $\times$ REM period interaction. The predicted STPM probabilities for a second set of CATMOD analyses are shown group $\times$ REM period $\times$ state in Table 4 to further illustrate this point. Though all three groups have a significant difference in destination state probability due to the effect of initial state, it appears that the DEPR group has, in addition, an effect due to REM period which approaches significance. The number of subjects gives insufficien power for this comparison, which is likely to be the cause of the significant group $\times$ REM period interaction effect of Table 3. (This interesting result suggests that if the study were re-done with a larger number of subjects, it might show that DEPR subjects have a different pattern of destination state probability over the REM periods of the night, whereas NORM and 


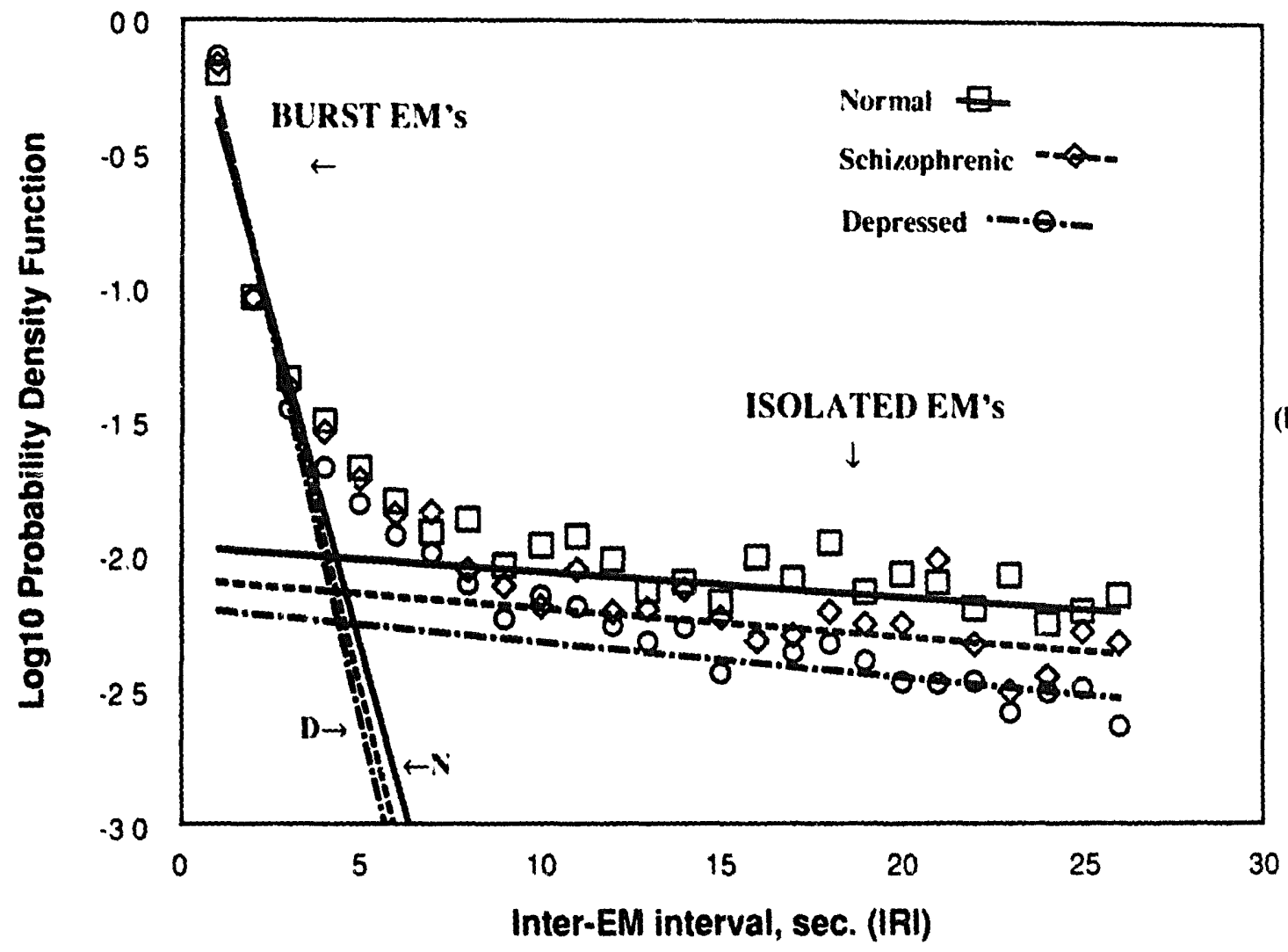

Figure 1.(b) PDFs using the two-process model of Boukadoum and Ktonas (1988). Simple linear regressions were fitted separately to burst and isolated IRI segments of the curves in (a). Burst fit was from 1 to $3 \mathrm{sec}$ IRI; isolated fit was from 15 to $25 \mathrm{sec}$ IRI. Regression equation $y=m X+b$ on semi-lng axes. Correlation is Pearson product-moment $r$.

Isol. fit: NORM $y=(-0.0092 X)-1.970 ; r^{2}=0.963$

SCHZ $y=(-0.0106 X)-2.093 ; r^{2}=0.964$

DEPR $y=(-0.0130 X)-2.192 ; r^{2}=0.971$

Burst fit: NORM $y=(-0.4895 X)+0.1159 ; r^{2}=0.961$

SCHZ $y=(-0.5458 X)+0.2410 ; r^{2}=0.962$

DEPR $y=(-0.5785 X)+0.2881 ; r^{2}=0.962$

The intersection of the burst and isolated functions for each group occurred at the following IRI (X-axis) points, in sec. This value is the best single choice (quantitization threshold) to separate burst from isolated IRIs.

$$
\begin{aligned}
& \text { NORM }=4.25 \\
& \mathrm{SCHZ}=4.36 \\
& \mathrm{DEPR}=4.38
\end{aligned}
$$

The same point as determined for normals by Boukadoum and Ktonas (1988) was $4.3 \mathrm{sec}$ IRI, although they used $5.00 \mathrm{sec}$ in their publication. We used $5.00 \mathrm{sec}$ as the threshold point in all our Markev calculatinne. for consistency with the above authors. 
SCHZ groups are stationary on such a measure.) The intercorrelation matrix of the conventional REM measures (Table 5) showed many large correlations, suggesting considerable redundancy in these variables. ANOVAs on a subset of these variables (Table 6) showed a significant excess of \#IRI and BURIRI/HR but no difference in \#MIN of REM sleep, in both depressed and schizophrenic groups compared with normals. Of particular interest was the ISOLIRI/HR, which did not differ between groups. This immediately suggests why the lines fitting the isolated IRI in Figure $1 \mathrm{~b}$ are approximately parallel but offset (see Discussion). Fragmentation was worst among depressed patients.

\section{Discussion}

To our knowledge, this is the first application of Markovian STPMs to the REM sleep of normals and psychiatric patients. Schizophrenic and depressed patients are clearly differentiated from normals; thus, the null hypothesis is rejected. However, the two patient groups are not easily distinguished from each other on absolute value, so the Markovian results parallel those of RD (Benson and Zarcone 1991).

A major limitation of the present study results from the DEPR group being significantly older than the SCHZ or NORM groups. Because young schizophrenics are compared with older depressed patients, there is no way of telling whether the results observed are due to age or diagnosis or both.

Our demonstration that the STPM is stationary over nights and REM periods in normals confirms the findings of Boukadoum and Ktonas (1988). We now extend this observation to schizophrenic and, provisionally, to depressed patients, although the latter were the only subjects to approach significant difference over REM periods. The STPM is indeed a consistent quantification of the phasic events of REM sleep. This is in direct contrast to EM density, which increases with successive REM periods during the night (Benson and Zarcone 1991). A practical implication is that a single REM period with over 20 EMs would suffice to calculate a valid estimate of an individual's characteristic $2 \times 2$ STPM, as the sampling error of the STPM decreases to a plateau at this point (Jansen and Cheng 1988).

Both the probability distribution analysis of IRIs and the Markovian analysis support a two-process model of the generation of eye movements in REM sleep, one process causing the emission of isolated IRIs of long duration, the other causing burst IRIs of very short duration. This explains why a simple negative exponential frequency distrifuriom, sing! puicus unuci, ciues not fit the observed data (Spreng et al 1968).

Our results also suggest some conclusions about the choice of parameters for STPM analysis. The linear regression of our NORM burst function in Figure $1 \mathrm{~b}$ has coefficients similar to that reported by Boukadoum and Ktonas (1988), despite the inclusion of about $20 \%$ more IRIs, and all in the $60-200 \mathrm{msec}$ range. This suggests that IRI emission in the rangc 60-200 msec is merely an extension of the function fitted to IRI greater than $200 \mathrm{msec}$, and need not always be excluded. Also, in future work of this type, $4.3 \mathrm{sec}$ rather than 5.00 suc would seem to be a more objective point at which to divide the burst from the isolated IRIs, as Boukadoum's normals and all three of our experimental groups showed threshold values very close to $4.3 \mathrm{sec}$.

What exactly is shown to be abnormal by our analysis that is not evident from older forms of phasic eveni analysis? The IRI probability distributions of Figure $1 \mathrm{~b}$ are striking in that the linear fits to the burst IRI distribution are virtually identical in the three experimental groups, whereas the fits to the isolated IRI distribution are parallel but offset 


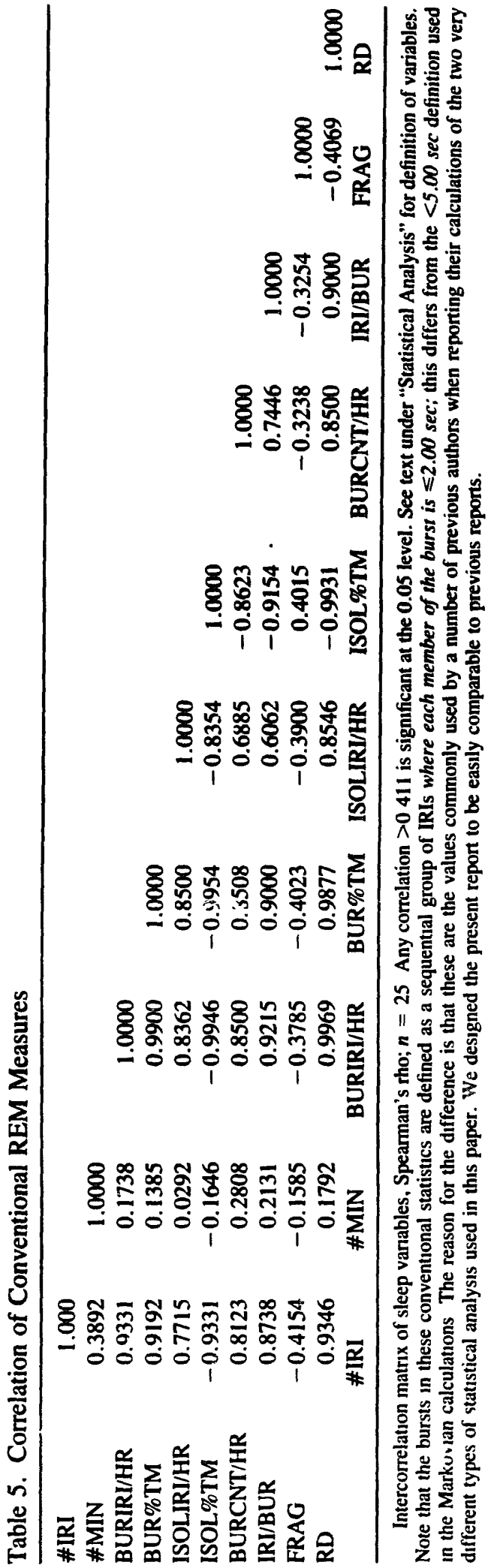


Table 6. ANOVA Using Selected Variables from Table 5

\begin{tabular}{|c|c|c|c|c|c|}
\hline Vanable & Source & df & $F$ & $p$ & Means \\
\hline \multirow[t]{5}{*}{ \#IP.I } & Group & 2 & 557 & 0.0047 & $\begin{array}{l}\text { NORM } 407 \\
\text { DEPR } 636^{a}\end{array}$ \\
\hline & & & & & $\mathrm{SCHZ} 686^{a}$ \\
\hline & REM period & 3 & 7.01 & 0.0002 & RP1 408 \\
\hline & & & & & RP3 $770^{a}$ \\
\hline & & & & & RP4 $616^{a}$ \\
\hline \multirow[t]{5}{*}{ \#MIN } & Group & 2 & 1.15 & N.S. & $\begin{array}{l}\text { NORM } 26.5 \\
\text { DEPR } 24.7\end{array}$ \\
\hline & & & & & SCHZ 29.0 \\
\hline & REM period & 3 & 7.78 & 0.0001 & RPI 20.5 \\
\hline & & & & & RP2 22.7 \\
\hline & & & & & RP4 26.5 \\
\hline \multirow[t]{7}{*}{ BURIRI/HR } & Group & 2 & 13.39 & 0.0001 & NORM 729 \\
\hline & & & & & DEPR $1405^{a}$ \\
\hline & & & & & $\mathrm{SCHZ} 1163^{a}$ \\
\hline & REM period & 3 & 1.69 & N.S. & RPI 947 \\
\hline & & & & & RP2 1056 \\
\hline & & & & & RP3 1166 \\
\hline & & & & & RP4 1128 \\
\hline \multirow[t]{6}{*}{ ISOLIRI/HR } & Group & 2 & 1.69 & N.S. & NORM 212 \\
\hline & & & & & DEPR 234 \\
\hline & & & & & SCHZ 232 \\
\hline & REM period & 3 & 2.51 & N.S. & RP1 221 \\
\hline & & & & & RP2 216 \\
\hline & & & & & RP3 218 \\
\hline \multirow{7}{*}{ FRAG } & & & & & \\
\hline & Group & 2 & 7.70 & 0.0007 & NORM $90.3^{a}$ \\
\hline & & & & & DEPR 80.1 \\
\hline & REM period & 3 & 2.38 & N.S & RPI 88.7 \\
\hline & & & & & RP2 88.4 \\
\hline & & & & & RP3 87.7 \\
\hline & & & & & RP4 81.3 \\
\hline
\end{tabular}

RPI = REM period number one, etc Three-way ANOVAs were initially performed, but the night variable was never ugmiticant. Accordingly, 2-way ANOVAs are reported here, pooling both nights together. SAS version 6.03 GLM procedure. Bonterroni post-hoc comparisons of means were done at the 0.95 confidence interval.

"Means that did not differ among themselves, but that differed significantly from other means in the same stratum.

considerably. This suggests a conclusion of theoretical importance: that the physiological process emitting the isolated IRIs is identical in NORM, DEPR, and SCHZ groups, the offset being due only to the greater number of IRIs in the patient groups (the divisor). In further confirmation, the bottom third of Table 2 shows no significant differences in the transition probability of isolated-to-isolated IRIs and Table 6 shows no significant group differences in the absolute number of these IRIs. 
The corollary is that the process that emits burst IRIs might be the source of the observed significant group differences. This is indeed the case. Whereas the probability distribution of the burst IRIs is very similar in all three groups, there are significant differences in the number of BURIRI/HR, as DEPR and SCHZ groups have nearly double the number found in NORM. The burst-to-burst transition probability (Table 2) is also significantly higher in the SCHZ and DEPR groups than NORM, whereas the isolated-to-burst and isolated-to-isolated transition probabilities do not differ between groups. This suggests that the entry into a new burst from a given isolated IRI is no more likely to occur in patients than normals. Once in the burst, however, the probability of the next IRI remaining in the burst is significantly higher in the SCHZ and DEPR groups versus NORM. Physiologically, this implies that the patients have an intact and normal burst IRI generator which is either being driven excessively by some other system, or which has lost some inhibitory input. The caveat regarding mean group ages applies to this conclusion also: it is possible that aging itself causes these effects.

A possible source of excess drive could be the vestibular system. Omitz et al (1973) demonstrated in normais that vestibular or auditory stimulation during REM sleep increased burst IRI activity. Alse there is a tenfold increase over preflight values in the number of burst IRIs when astronauts sleep in the weightless conditions of earth orbit (Petre-Quadens and Dequae 1987).

Biochemically, some preliminary data (Douglass et al 1930) suggest that the M: muscarinic (cholinergic) system might be involved in the overactive burst generator in the patients. The M1 antagonist biperiden failed to alter the Markovian probabilities in normals, but in schizophrenics it reduced the abnormally high burst-to-burst transition probability while leaving the isolated-to-isolated transition probability unchanged. Biperiden had a similar effect on RD, lowering it in schizophrenics but not altering it in normals. This dissociation of the effect of M1 blockade on burst versus isolated IRI production suggests that excess burst IRIs in schizophrenics are controlled by M1 cholinergic systems, although M2 pontine systems seem to control the onset of the REM state as a whole. This is in accord with Hobson's (1990) observation of the effect of cholinomimetic drugs applied directly to the pons in the cat, wherein the FGO (pontinegeniculate-occipital) burst cells of the region were stimulated to produce PGO waves. PGO waves have not been observed in the human, as they are usually measured by implanted brain electrodes. They are believed to be the cause of the rapid eye movements of REM sleep.

The present findings suggest that the Markovian burst-to-burst transition probability might be a reflection of the activity of the PGO-wave-generating cells of the peribrachial pons. The reason why this activity should be increased in schizophrenia and depression is not yet clear, but it seems to involve 5HT and cholinergic mechanisms. Benson et al (1983) found an inverse correlation between cerebrospinal fluid levels of the serotonin metabolite 5-HIAA and burst EM measures in psychiatric patients. Combining this observation with the biperiden data suggests the possibility of an imbalance between cholinergic and serotonergic systems in schizophrenics and depressives as the cause of the increased burst-to-burst transition probability. This hypothesis should be amenable to pharmacological testing in humans and animals.

This research was supported in part by a Research Fellowshıp to Dr. Douglass from the Alberta Heritage Foundation for Medical Research, and by the following: the Medical Research Service of the Department of Veterans Affairs; a grant from the VA Medical Research Service to the Schizophrenia Biologic Research Center 
at the Palo Alto VA Medical Center; NIMH Grants MH30854 and MH37252; and a grant from the John D. and Catherine T. MacArthur Foundation. The autnors thank the staff of the Stanford-VA Mental Health Clinical Research Center for their help in this investigation

\section{References}

Benson KL, Zarcone VP Jr (1989): Slow wave sleep deficits: Their magnitude, distribution, and reliability in schizophrenia and major depressive disorder. Biol Psychiatry 25:202A.

Benson KL, Zarcone VP Jr (1991): REM sleep eye movements in schizophrenia and depression. Arch Gen Psychiatry (in press).

Benson KL, Zarcone VP Jr, Faull KF, Barchas JD, Berger PA (1983): REM sleep eye movement activity and CSF concentrations of 5-hydroxy-indoleacetic acid in psychiatric patients. Psychiatry Res 8:73-78.

Bishop YMM, Fienberg SE, Holland PW (1975): Discrete Multivariate Analysis: Theory and Practice. Cambridge, MA: The MIT Press.

Boukadoum AM (1983): Rapid Eye Movement (REM) Occurrence Patterns During Stage I-REM Sleep in Humans: An Automated Study Using Markovian Modeling. Ph.D. Thesis, University of Houston. Ann Arbor MI: University Microfilms International.

Boukadoum AM, Ktonas PY (1986): EOG-based recording and automatic detection of sleep rapid eye movements: A critical review, and some recommendations. Psychophysiology 23(5):598611.

Boukadoum AM, Ktonas PY (1988): Non-random patterns of REM occurrences during REM sleep in normal human subjects: An automated second-order study using Markovian modeling. EEG Clin Neurophysiology 70:404-416.

Douglass AB, Benson KL, Zarcone VP Jr (1985): The frequency distribution of rapid eye movements in normal, depressed, and schizophrenic subjects (abstract). Sleep Res 14:127.

Douglass AB, Tandon R, Shipley JE, Taylor S, Goodson J (1990): REM density changes in schizophrenia due to biperiden (abstract). Biol Psychiatry 27:108A.

Feinberg 1, Koresko RI, Gottlieb F (1964): Further observations on electrophysiological sleep patterns in schizophrenia. Compr Psychiatry 6(1):21-24.

Foster FG, Kupfer DJ, Coble P, McPartland RJ (1976): Rapid eye movement sleep density: An objective indicator in severe medical-depressive syndromes. Arch Gen Psychiatry 33:11191123.

Gillin JC, Wyatt RJ (1975): Schizophrenia: Perchance a dream? Int Rev Neurobiology 17:297342.

Hobson JA (1990): Sleep and dreaming. J Neuroscience 10(2):371-382.

Hord D (1975): Common-mode rejection techniques in conjugate eye movement recording during sleep. Psychophysiology 12:354-355.

Jansen BH, Cheng WK (1988): Structural EEG analysis: An explorative study. Int J Biomed Comput 23(3-4):221-237.

Kemp B, Kamphuisen HAC (1986): Simulation of human hypnograms using a Markov chain model. Sleep 9(3):405-414.

Kempenaers C, Kerkhofs M, Linkowski P, Mendlewicz J (1988): Sleep EEG variables in young schizophrenic and depressive patients. Biol Psychiatry 24:828-833.

Keshavan MS, Reynolds CF, Kupfer DJ (1990): Electroencephalographic sleep in schizophrenia: A critical review. Comp Psychiatry 30(1):34-47.

Krynicki V (1975): Time trends and periodic cycles in REM sleep eye movements. EEG Clin Neurophysiology 39:507-513. 
Ktonas PY (1974): Semiautomatic Detection and Analysis of REM Patterns in Human Sleep. PhD Thesis, University of Florida. Ann Arbor, MI: University Microfilms International.

Ktonas PY, Bonilla JF (1979): Quantification of time connectivity patterns in point processes. In IEEE 1979: Frontiers of Engineering in Health Care. New York: IEEE, pp. 246-249.

Ktonas PY, Smith JR (1976): Semi-automatic analysis of rapid eye movement (REM) patterns: A software package. Comput Biomed Res 9:109-124.

Ktonas PY, Bo:kkadoum AM (1987): Recording, automated detection, and quantification of rapid eye movement (REM) patterns during human REM sleep. In Scheving LE, Halberg F, Ehret CF (eds), Proceedings of the NATO Advanced Research Workshop on "Chronobiotechnology and Chronobiological Engineering." Boston: Martinus Nijhoff Publishers, pp. 229-240.

Ktonas PY, Bonilla JF, Boukadoum AM (1981): Quantification of time-connectivity patterns in rapid eye movement occurrences during sleep. IEEE Trans Biomed Engineering 28(1) 31-36.

Kupfer DJ (1976): REM latency: A psychobiologic marker for primary depressive disease. Biol Psychiatry 11(2):159-174.

Kupfer DJ, Ulrich RF, Coble PA, et al (1984): Application of automated REM and slow-wave slecp analysis II: Testing the assumptions of the two-process model of sleep regulation in normal and depressed subjects. Psychiatry Res 13:335-343.

Lavie P (1979): Rapid eye movements in REM sleep-more evidence for a periodic organization. EFG Clin Neurophysiology 46(6):683-688.

Ornitz EM, Forsythe AB, de la Pena A (1973): The effect of vestibular and auditory stimulation on the rapid eye movements of REM sleep in normal children. EEG Clin Neurophysiology 34:379-390.

Petre-Quadens O, Dequae PA (1987): The synergetics of the eye movements of REM sleep. In Scheving LE, Halberg F, Ehret CF (eds), Proceedings of the NATO Advanced Research Workshop on "Chronobio-Technology and Chronobiolugical Engineering." Boston: Martinus Nijhoff Publishers, pp 216-228.

Rechtschaffen A, Kales A (eds) (1968): A Manual of Standardized Terminology Technigues ard Scoring System for Sleep Stages of Human Subjects. National Institute of Health Publication No. 204. Washington: U. S. Government Printing Office.

Reich L, Weiss BL, Coble P, McPartland R, Kupfer DJ (1975): Sleep disturbance in schizophrenia: A revisit. Arch Gen Psychiatry 32:51-55.

Reynolds CF, Christiansen CL, Taska LS, Coble PA, Kupfer DJ (1983): Sleep in narcolepsy and depression. Does it all look alike? J Nerv Ment Dis 171(6):290-295.

Schreier L, Rule RB, Benson K, Zarcone V (1977): REMDTEK: An interactive software package for the detection and quantification of rapid eye movements of stage REM (abstract). Sleep Res 6:214.

Snedecor GW, Cochran WG (1980). Statistical Methods (ed 7). Ames, Iowa: Iowa State University Press.

Spitzer RL, Endicott J, Robins E (1978): Research Diagnostic Criteria (RDC) for a Selected Group of Functional Disorders. New York: Biometrics Research, New York State Psychiatric Institute.

Spreng LF, Johnson LC, Lubin A (1968): Autonomic correlates of eye movement bursts during stage REM sleep. Psychophysiology 4(3):311-323.

Thase ME, Kupfer DJ, Ulrich RF (1986): Electroencephalographic sleep in psychotic depression. Arch Gen Psychiatry 43:886-893.

Zarcone VP Jr, Benson KL (1983): Increased REM eye movemunt density in self-rated depression. Psychiatry Res 8:65-71.

Zarcone VP, Benson KL, Berger PA (1987): Abnormal rapid eye movement latencies in schizophrenia. Arch Gen Psychiatry 44(1):45-49. 